\title{
There is Nothing More Flattering
}

Andrew J. McLean ${ }^{1}$

Received: 8 February 2016 / Accepted: 30 March 2016/Published online: 10 May 2016

(C) Academic Psychiatry 2016

There is nothing more flattering Than being useful and forgettableHearing a paraphrase

Of one's words from times past Coming out of another's mouth As if they were their own.

It is the legacy of

A capable teacher.

Acknowledgement

Is simply icing on the cake.

$\triangle$ Andrew J. McLean

andrew.mclean@med.und.edu

1 University of North Dakota School of Medicine and Health Sciences, Fargo, ND, USA 